\title{
Context-Aware Delivery of Information in Assistive Environments utilizing Future Internet Technologies
}

\author{
Charalampos Doukas \\ University of the Aegean \\ Samos, Greece \\ doukas@aegean.gr
}

\author{
Nikos Fotiou \\ Athens University of \\ Economics and Business \\ Athens, Greece \\ fotiou@aueb.gr \\ Ilias Maglogiannis \\ University of Central Greece \\ Lamia, Greece \\ imaglo@ucg.gr
}

\author{
George C. Polyzos \\ Athens University of \\ Economics and Business \\ Athens, Greece \\ polyzos@aueb.gr
}

\begin{abstract}
In this paper, we investigate the potentials of an architecture for enabling the context-aware content adaptation and specialized delivery of information, based on a Future Internet architecture; the Publish/Subscribe Internetworking (PSI or $\Psi$ for short) architecture. $\Psi$ is an information oriented architecture built for the Future Internet using the so-called publish/subscribe paradigm. We advocate that by bringing information in the center of our solution as well as by applying $\Psi$ 's particular security features we can achieve seamless information morphing and effective access control policies
\end{abstract}

\section{Categories and Subject Descriptors}

J.3 [Life and Medical Sciences]: Medical information systems; H.m [Information Systems]: Miscellaneous

\section{General Terms}

Design, Experimentation, Standardization, Theory

\section{Keywords}

Content adaptation, Context awareness, Assistive information delivery, Future Internet

\section{INTRODUCTION}

The introduction of pervasive healthcare paradigm has enabled the awareness towards the elderly and the need for constant medical supervision of chronic patients or habitants at remote, isolated and underserved locations. In this context, advanced electronic healthcare services that were once available at big hospitals, are now required to be made available through a network anytime, anyplace and to anyone. A

Permission to make digital or hard copies of all or part of this work for personal or classroom use is granted without fee provided that copies are not made or distributed for profit or commercial advantage and that copies bear this notice and the full citation on the first page. To copy otherwise, to republish, to post on servers or to redistribute to lists, requires prior specific permission and/or a fee.

PETRA '11 May 25 - 27, 2011, Crete, Greece

Copyright 2011 ACM ISBN 978-1-4503-0772-7/11/05 ...\$10.00. medical assistive environment on the other hand concerns the utilization of pervasive and ubiquitous technologies for delivering the above services. The word "assistive" may also be used in a general way, including not only assisting persons with obvious health problems, but also empowering any human to improve the quality of life by enhancing sensing and computer based support at all levels. Thus the type of information that is exchanged in assistive environments is characterized by diversity and thus tools are needed for content adaptation.

In such an environment, the notion of Future Internet and the new concepts/architectures that brings with, such as distributed and ad-hoc networking, cloud computing and elasticity in resources, Internet of Things and many others, can fit perfectly, enabling the development of advanced assistive applications. In this work we discuss the application of Future Internet technologies and architectures in assistive environments focusing on advanced networking and context awareness issues. We are particularly focused on a clean slate information oriented architecture; the $\Psi$ architecture. $\Psi$ is a Future Internet architecture which utilizes information as its building block. It is based on the so-called publish/subscribe paradigm and it is designed to support effective and flexibly information governance and dissemination. Information centric architectures appear to be ideal for pervasive healthcare environments as they achieve effective information collection, provision, processing and governance [11].

The rest of the paper is structures as follows: Section 2 presents the adaptation and context awareness requirements in assistive environments, while Section 3 summarizes the Future Internet technologies that can be exploited. Section 4 describes a proposed Future Internet architecture that creates an assistive environment and Section 5 presents future work and concludes the paper.

\section{INFORMATION DELIVERY AND CON- TEXT AWARENESS IN ASSISTIVE EN- VIRONMENTS}

The success of an assistive environment relies on the proper, accurate and on time delivery of information and data exchange between the involved individuals (users and operators). The latter however in addition to the complexity 
of assistive environments (different devices, data types, user requirements, different medical personnel involved, etc.) introduces several issues that need to be addressed:

1. Assistive environments have special requirements on content and information presentation (e.g., case of the elderly and people with vision impairment)

2. Different presentation layers exist. For instance, patient data has to be coded in different formats for transmission and decoded respectively for a medical expert to assess it. Different device specifications also introduce issues in data interoperability for both collecting and transmitting various contextual data. Moreover the access network of a user may be the bottleneck of a rich data transmission session.

3. Specialized delivery in context of reactive/proactive data transmission (cases of emergency, etc.) is needed.

4. The transmitted information usually contains sensitive data that needs to be secured. Patient data should be protected from eavesdropping as well as from manipulation during the transmission.

The following sections provide information on context awareness in assistive environments and present the proposed platform.

Context awareness is the capability of the networking applications to be aware of the existence and characteristics of the user's activities and environments. In rapidly changing scenarios, such as the ones considered in the fields of mobile, pervasive, or ubiquitous computing, systems have to adapt their behavior based on the current conditions and the dynamicity of the environment they are immersed in [7]. A system is context-aware if it can extract, interpret and use context information and adapt its functionality to the current context of use. The challenge for such systems lies in the complexity of capturing, representing and processing contextual data. In assistive environments, contextual information might refer to user context (e.g., special user requirements like nutrition, etc., user status and location, etc.), the environment context (e.g., indoor or outdoor), and the hardware context (presentation and communication devices used, network status, etc.). To capture context information generally some additional sensors and/or programs are required [1].

The way context-aware applications make use of context can be categorized into the three following classes: presenting information and services, executing a service, and tagging captured data.

- Presenting information and services refers to applications that either present context information to the user, or use context to propose appropriate selections of actions to the user.

- Automatically executing a service describes applications that trigger a command, or reconfigure the system on behalf of the user according to context changes.

- Attaching context information for later retrieval refers to applications that tag captured data with relevant context information.

One way to capture and distribute contextual data is through the use of intelligent agents. Intelligent agents can be viewed as autonomous software (or hardware) constructs that are proactively involved in achieving a predetermined task and at the same time reacting to its environment. According to [4], agents are capable of:

- Performing tasks (on behalf of users or other agents).

- Interacting with users to receive instructions and give responses.

- Operating autonomously without direct intervention by users, including monitoring the environment and acting upon the environment to bring about changes.

- Showing intelligence-to interpret monitored events and make appropriate decisions.

Agents can be proactive, in terms of being able to exhibit goal-directed behavior, reactive; being able to respond to changes of the environment, including detecting and communicating to other agents, autonomous; making decisions and controlling their actions independent of others. Intelligent agents can be also considered as social entities where they can communicate with other agents using an agentcommunication language in the process of carrying out their tasks.

In the context of pervasive healthcare, intelligent agents can contribute by analyzing patient and contextual information, distributing tasks to responsible individuals, informing users regarding special actions and circumstances. Decision making is performed usually through data classification on the acquired patient signals and contextual information. Generated classification results can contain information concerning the status of a patient, suggested diagnosis, behavioral patterns, etc. Data classification is important problem in a variety of engineering and scientific disciplines such biology, psychology, medicine, marketing, computer vision, and artificial intelligence [12]. Its main object is to classify objects into a number of categories or classes. Depending on the application, these objects can be images or signal waveforms or any type of measurements that need to be classified. Given a specific data feature, its classification may consist of one of the following two tasks: a) supervised classification in which the input pattern is identified as a member of a predefined class; b) unsupervised classification in which the pattern is assigned to a hitherto unknown class.

In statistical data classification, input data are represented by a set of $n$ features, or attributes, viewed as a $\mathrm{n}$-dimensional feature vector. The classification system is operated in two modes: training and classification. Data preprocessing can be also performed in order to segment the pattern of interest from the background, remove noise, normalize the pattern, and any other operation which will contribute in defining a compact representation of the pattern. In the training mode, the feature extraction/selection module finds the appropriate features for representing the input patterns and the classifier is trained to partition the feature space. The feedback path allows a designer to optimize the preprocessing and feature extraction/selection strategies. In the classification mode, the trained classifier assigns the input pattern to one of the pattern classes under consideration based on the measured features.

There is a vast array of established classification techniques, ranging from classical statistical methods, such as 
linear and logistic regression, to neural network and treebased techniques (e.g., feed-forward networks, which includes multilayer perception, Radial-Basis Function networks, Self-Organizing Map, or Kohonen-Networks), to the more recent Support Vector Machines. Other types of hybrid intelligent systems are neuro-fuzzy adaptive systems which can comprise of an adaptive fuzzy controller and a network-based predictor. More information regarding data classification techniques can be found in [12].

The following section presents the proposed architecture that enables the context awareness and content adaptation in assistive environment.

\section{A FUTURE INTERNET ARCHITECTURE}

The current Internet architecture has been evolved around the so- called end-to-end principle; it was designed based on the need of interconnecting efficiently mainframes and minicomputers and to provide efficient remote access to them. However with the Internet evolution information items rather than remote machines, become the main users's interest nevertheless the core design of the Internet remained unchanged. The end-to-end design principle of the current Internet architecture has been identified as the root cause of many of its limitations, including lack of effective mobility and multicast support as well as lack of security, quality of services and economics [3]. Various add-ons, such as NATs, Mobile IP, CDNs, p2p overlays, etc., all violate, in various ways, several aspects of the original Internet architecture in order to provide answers to features that were not part of the original design (or the original requirements). With all these in mind various research efforts were launched in order to redesign the Internet architecture. In the rest of this paper we will consider as reference architecture for the Future Internet the $\Psi$ architecture. The $\Psi$ architecture is an ongoing research effort for creating a clean slate, information oriented architecture for the Future Internet started with the FP7 PSIRP ${ }^{1}$ project and being continued with the FP7 PURSUIT ${ }^{2}$ project.

The $\Psi$ architecture considers nothing for granted-not even IP-it rather applies a complete different communication paradigm in all the layers of the architecture; the Publish/Subscribe (Pub/Sub) paradigm. Architectures that abide by this paradigm are mainly composed by three entities; the publishers, the subscribers and an event notification service [2]. Publishers are content producers that advertise and publish the content they own. Subscribers are content consumers, who express their interest for specific content by issuing subscription messages. The event notification service is responsible for matching the advertised content with subscribers' interest and for initiating a content forwarding process from publishers towards subscribers. The pub/sub paradigm allows for endpoints decoupling as publication and subscription operations do not need to be synchronized. This decouple offers significant security advantages and it facilitates the deployment of mobility and multicast mechanisms. Moreover the pub/sub paradigm allows the existence of multiple publishers or a specific publication, enabling this way caching and multi homing [6].

Information is the building block of the $\Psi$ architecture; in-

\footnotetext{
${ }^{1}$ http://www.psirp.org

${ }^{2}$ http://www.fp7-pursuit.eu
}

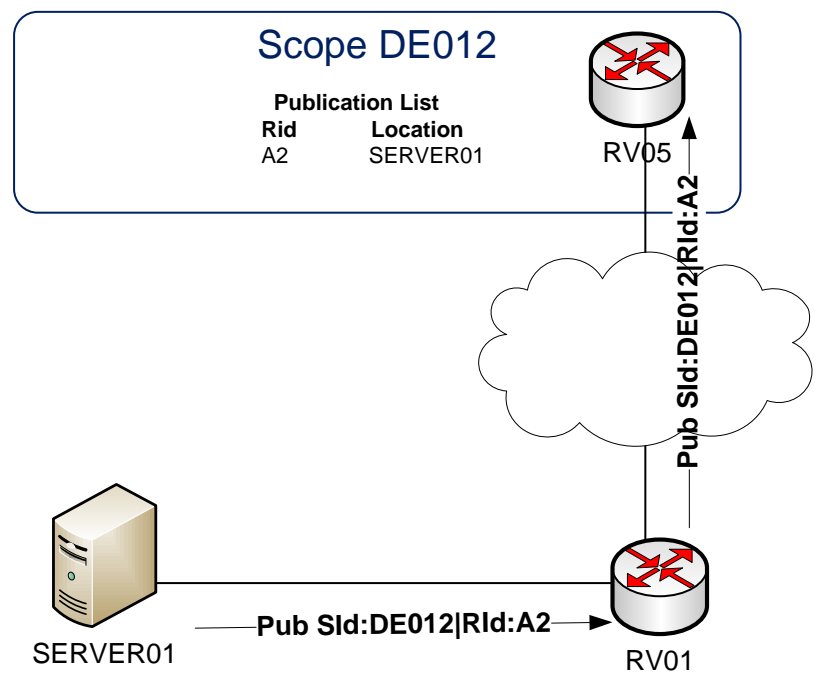

Figure 1: Publication in the $\Psi$ architecture

formation is everything and everything is information [10]. Every information item in $\Psi$ is identified by a unique flat identifier named the Rendezvous Identifier (RId). Information items are organized in scopes. Scopes are physical or logical structures hierarchically organized that are used to locate information items as well as to control their dissemination. A corporate network is an example of a physical scope whereas a social network is an example of a logical scope. Every scope is governed by dissemination policies which dictate who can publish information in this scope as well as who can subscribe to a scope's information items. Every scope is identified by a unique flat identifier named the Scope Identifier (SId) and is managed by-at least-one network entity called the Rendezvous Node (RV). All RVs are organized in a network known as the rendezvous network. The rendezvous network enables scope lookup and it can be hierarchically organized (akin to DNS) or flat (such as DHTs). An information item may belong to multiple scopes. In order for a publisher to advertise an information item he posses, he has to specify the item's RId as well as the SId of the scope that will be responsible for this information item. The RId of an information item is application specific; it can be for example the result of the application of a hash function over the item's data. Similarly in order for a subscriber to receive an information item, a subscription message has to be issued towards the RV of the scope in which the information item belongs, this RV becomes the so-called Rendezvous Point for this information item. Every publisher knows at least one RV which is his default RV. So at it can be seen in Figure 1, the publisher SERVER01 sends the publication message to its default RV which routes it to the appropriate RV. In Figure's 1 case the publisher wants to publish an information item to scope DE012, which is managed by RV05; it sends the publication message to his default RV, i.e., RV01, and it eventually reaches RV05. When the publication message reaches the RV05 the publication list of the scope is updated. The subscriber therefore needs to know the SId of the scope and the RId of the information item. SIds and RIds can be learned by an out-of-band mechanism, such as a search engine. Upon receiving a suc- 


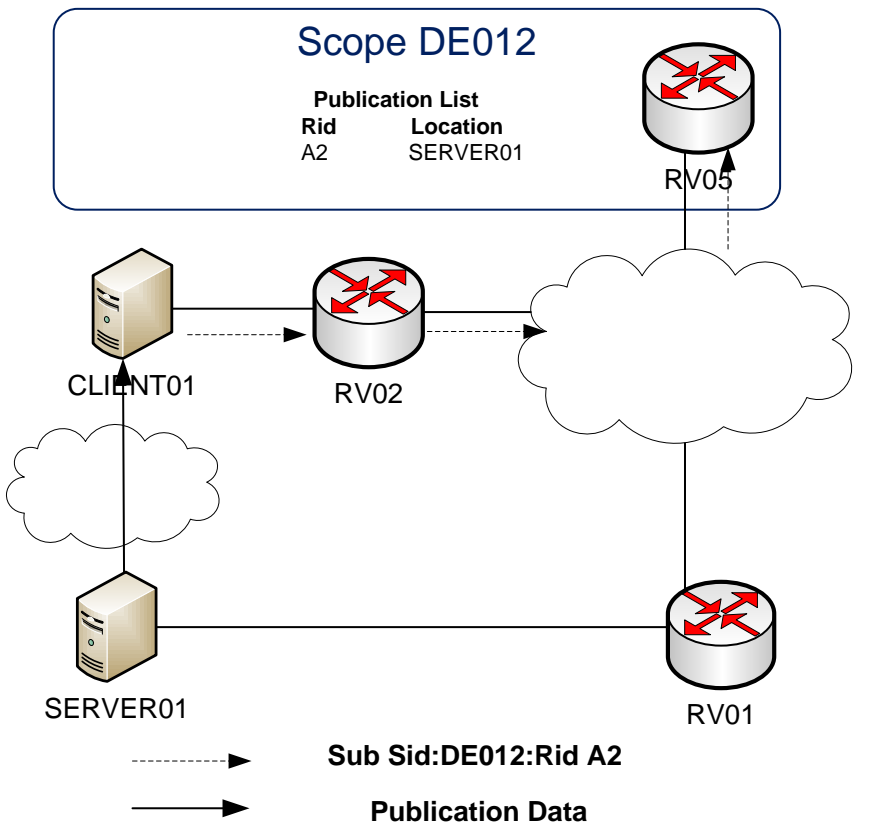

Figure 2: Subscription in the $\Psi$ architecture

cessful subscription the RV initiates a (multicast) delivery path creation process from publisher towards subscriber(s).

This process is achieved by utilizing information accumulated by a network of Topology Managers (TMs) and by taking into account subscriber's requirements as well as possibly delivery policies. TMs are network nodes responsible for monitoring the network topology and for detecting topology changes. In order for an information item to be forwarded through a specific delivery path a bloom filter based structure is used, known as the zFilter [5]. zFilters contain all the links that an information item needs to traverse in order to reach its destination(s). The zFilter formation process makes difficult for a malicious entity to manipulate a delivery path.

Figure 2 shows a subscription example. Subscriber CLIENT01 subscribes to the already published information item with RId A2 in scope DE012. When the subscription message reaches RV05 a forwarding path is created between SERVER01-which is the publisher- and CLIENT01. Finally the publication is forwarded from SERVER01 to CLIENT01.

The $\Psi$ architecture seeks to secure the information itself rather than the communication channel. Therefore information can be delivered even over unsecured channels. Scopes enable information access control, as policies can defined over who can publish information as well as who can subscribe to published items. Information transmissions are secured using Packet Level Authentication (PLA) mechanisms that guarantee the confidentiality and the integrity of the transmitted items. PLA can also be used as an accountability mechanism [8] in case of a misbehaving entity. In the higher levels of the architecture existing security solutions can be applied with minor modifications [9]. The pub/sub paradigm deployed by the $\Psi$ architecture offers a significant level of protection against (Distributed) Denial of Service (DDoS) attacks and Spam communication as there is no information flow as long as the receiver has not ex- pressed his interest on a particular piece of information, i.e., the receiver in a pub/sub architecture is able to instruct the network which pieces of information shall be delivered to him. Moreover no information is requested from a publisher, unless the publisher has explicitly denote its availability, i.e., unless the publisher has advertise a publication for this particular piece of information. Finally the pub/sub paradigm enables the development of anonymization mechanisms as publication and subscription operations are decoupled and it is able to achieve better network availability through the deployment of caching, multicast and multihoming.

\section{THE PROPOSED ARCHITECTURE}

In this section we describe an architecture that creates an assistive environment overlaid over a $\Psi$ network. The purpose of this architecture is twofold; by continually monitoring the user's health status it delivers him useful information, including doctor's prescription, medical videos, medication alerts and in case of an emergency it provides immediate proactive information to medical personnel and alert the responsible individuals.

\subsection{Architecture components}

The architecture is composed by Storage Devices, Patient Monitoring Equipment, Patient's Visualization Equipment and Content Transformation Nodes. All these entities are interconnected over a $\Psi$ underlay network.

The Storage Devices are network nodes that are used to store big amount of data, including users medical records, medical images, informative videos, doctor's contact information etc. They have big storage capacity and they achieve availability through replication.

The Patient's Monitoring Equipment is composed by devices that monitor patient's health status as well as his context. Such devices are sensors deployed in patient's body, cameras, microphones medical equipment etc. These devices are associated with the identity of a specific patient using a variety of identification techniques ranging from usernamepassword login to smart cards or even biometrics.

The Patient's Visualization Equipment is the equipment used by patient's in order to view various information items such as doctor's instructions, informative videos, their health status etc. This equipment can be composed of various devices of different capabilities, such HDTVs, PCs, PDAs and mobile phones.

The Content Transformation Nodes are network elements that are able to transform various content types, for example they are able to transcode a video, resize an image or remove image from a video and transform it to an audio file.

All these nodes act as publishers and subscribers in a $\Psi$ underlay network. The $\Psi$ network is composed by the Rendezvous Network and the network of Topology Managers. The Rendezvous Network is the network responsible for the architecture's core functionality, as it enables information location, creation of delivery paths and the application of access control mechanisms. Topology Managers on the other hand are these network nodes that provide feedback to the Rendezvous Network regarding the topology status. Such feedback can be for example, that a user is connected to the architecture through a mobile network.

\subsection{Functions}




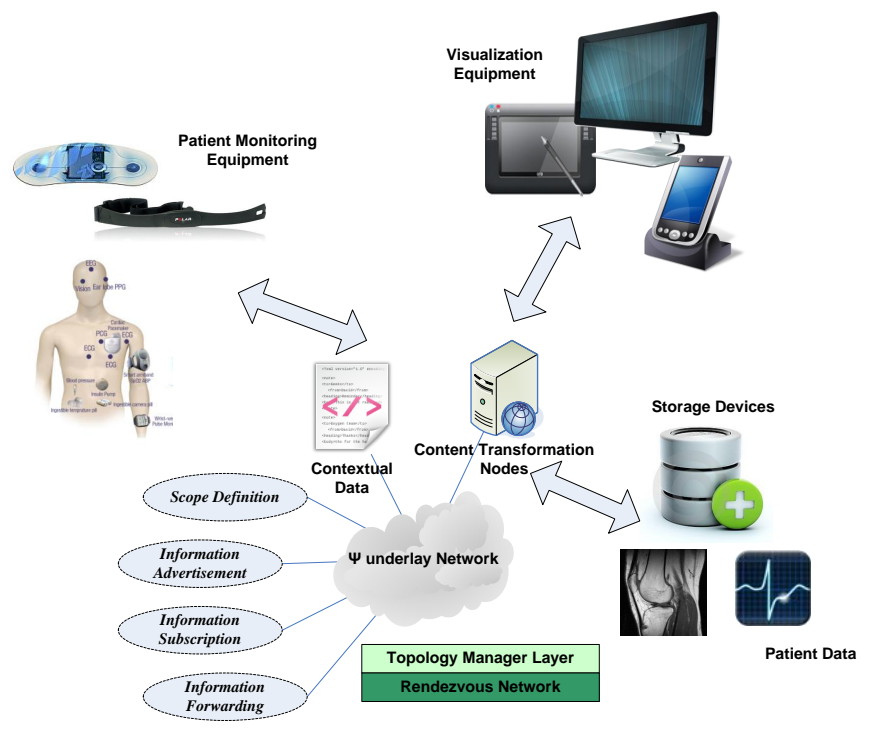

Figure 3: Main Architecture components of the proposed platform

The basic functions of the architecture are: Scope Definition, Information Advertisement, Information Subscription and Information Forwarding.

With the Scope Definition function scopes are defined. Each scope has its own dissemination strategy which is defined using an attribute based access control scheme. Access on the content stored in each scope is based on users' attributes as well as on their context. Scopes can be of general purpose, e.g., "a scope that provides information regarding an illness" or special purpose, e.g., "a scope that provides information regarding patient's X health". For the former general purpose access rules can be used, such as "all doctors can publish, all users can subscribe" whereas for the latter fine grained policies have to defined, such as "only devices of patient X can publish, only doctor Y can subscribe". A scope's policy can be changed upon need by taking into consideration user's context. For example the policy of a scope that provides the medical records of patient $\mathrm{X}$ can be "if the health status of patient $\mathrm{X}$ is normal, only doctor $\mathrm{Y}$ can subscribe, in case of emergency the relatives of $\mathrm{X}$ as well as doctors that are located inside hospital $\mathrm{M}$ can subscribe". A node in the rendezvous network is chosen in order manage a scope. Although rendezvous nodes do not store the actual content they shall implement all these complex policies, which include continuous input from users' devices.

With the Information Advertisement function the various components of the architecture advertise the information that generate. This information is advertised to the appropriate scope, i.e., to the scope which best reflects the desired dissemination strategy. Information advertisement contains an information identifier that will be used for referring to this information item, information item's location, i.e., the location of the storage node in which this item has been stored, publishers' credentials and context information as well as metadata information such as information mime type, author, size, expiration time, description etc. The advertisement message is send to the node of the rendezvous network that manages the desired scope. In order make an information item available to multiple scopes, multiple advertisements have to be sent to the respective rendezvous nodes. Upon receiving an information advertisement message the rendezvous node uses publishers' credentials and his context information in order to validate that he is eligible to publish information under this scope.

With the Information Subscription access to an information item is requested. A subscription can have many forms; it can be for example a subscription for a specific information item such as "an X-Ray image" or a subscription for a collection of items such as "medical advices from my doctor". Information Subscription and Information Advertisement functions are decoupled, therefore subscriptions for content that has not yet been generated can be send. For example a patient may subscribe to messages that notifies him that is time to take his medicine, or a doctor may subscribe for messages that notifies him that one of his patients is in emergency situation. It should be noted here that all these notification messages are information items also generated and advertised by the appropriate users/devices to the appropriate scope with the appropriate ID. As an example a scope "patient X" can be defined with SId "G235" and it can be agreed that all request for help are published in this scope with identifier "H2EF21", all the persons that are interested in receiving "request for help" messages should send a subscription for H2EF21 to the rendezvous node responsible for managing G235. The Information Subscription message contains the identifier of the information item to which the user wishes to subscribe (wildcards can also be used), user's credentials and context information as well as various metadata information such as desired content format, desired content type etc. User's credentials and context information are used by the rendezvous node in order to validate that the subscriber is legitimate to access the information item in question.

Information if forwarded using the Information Forwarding function. Information is forwarded upon a successful subscription/advertisement match which triggers the creation of a forwarding path from information storage location towards the subscriber(s). The path creation is implemented using information provided by the Topology Managers and it has as input the subscriber's requirements and context. Subscriber's requirements may include content adaptation for specific networks and/or devices. In this case the forwarding path will include Content Transformation Nodes. Information Forwarding can be reliable or unreliable, depending on the content type transferred as well as subscriber's needs.

\section{DISCUSSION AND CONCLUSION}

Context-aware assistive environments face difficulties due to the limitations of the current Internet design. Such systems demand complex information manipulation and effective security mechanisms which currently have to be provided with add-on solutions. This is necessary as users utilize various devices for creating and retrieving information as well as because the generated content contains sensitive data which has to be properly secured.

In this paper we presented a conceptual solution for a context-aware assistive environment based on a Future Internet architecture; the $\Psi$ architecture. We showed that by designing an architecture around information items and by providing functions for manipulating and securing them, pervasive health assistive solutions can be easier deployed. 
In the Future Internet it is expected that functionality currently implement by add-on solutions, will be part of the architecture's design. The solution presented enables information morphing based on users' requirements, proactively request for information items as well as through the notion of scopes it provides an access control mechanism.

The $\Psi$ architecture is under development. An open source prototype has already been provided and a testbed spanned across the Europe has been set up. We believe that the implementation and the evaluation of our conceptual architecture is possible and our future work is towards this direction.

\section{ACKNOWLEDGMENTS}

The work reported in this paper was supported by the FP7 ICT project PURSUIT, under contract ICT-2010-257217

\section{REFERENCES}

[1] C. Doukas, I. Maglogiannis, and G. Kormentzas. Advanced telemedicine services through context-aware medical networks. In International IEEE EMBS Special Topic Conference on Information Technology Applications in Biomedicine. IEEE, 2006.

[2] P. T. Eugster, P. A. Felber, R. Guerraoui, and A.-M. Kermarrec. The many faces of publish/subscribe. ACM Comput. Surv., 35:114-131, June 2003.

[3] A. Feldmann. Internet clean-slate design: what and why? SIGCOMM Comput. Commun. Rev., 37:59-64, July 2007.

[4] J. Fox, M. Beveridge, and D. Glasspool. Understanding intelligent agents: analysis and synthesis. AI Commun., 16:139-152, August 2003.

[5] P. Jokela, A. Zahemszky, C. Esteve Rothenberg, S. Arianfar, and P. Nikander. Lipsin: line speed publish/subscribe inter-networking. In Proceedings of the ACM SIGCOMM 2009 conference on Data communication, SIGCOMM '09, pages 195-206, New York, NY, USA, 2009. ACM.

[6] K. Katsaros, G. Xylomenos, and G. Polyzos. A hybrid overlay multicast and caching scheme for information-centric networking. In INFOCOM IEEE Conference on Computer Communications Workshops, 2010, pages 1-6. IEEE, 2010.

[7] K. K. Khedo. Context-aware systems for mobile and ubiquitous networks. Mobile Communications and Learning Technologies, Conference on Networking, Conference on Systems, International Conference on, 0:123, 2006.

[8] D. Lagutin and S. Tarkoma. Cryptographic signatures on the network layer-an alternative to the ISP data retention. In The IEEE symposium on Computers and Communications, pages 87-93. IEEE, 2010.

[9] P. Nikander and G. Marias. Towards understanding pure publish/subscribe cryptographic protocols. In Sixteenth International Workshop on Security Protocols. Cambridge, England, 2008.

[10] Tarkoma, S., ed. PSIRP deliverable 2.3, architecture definition, component descriptions, and requirements (d2.3), March 2010. http://www.psirp.org/.

[11] D. Trossen, D. Pavel, K. Guild, J. Bacon, and J. Singh. Information-centric pervasive healthcare platforms. In Pervasive Computing Technologies for
Healthcare (PervasiveHealth), 2010 4th International Conference on, pages 1-4. IEEE.

[12] J. Zhai, S. Zhang, and X. Wang. An Overview of Pattern Classification Methodologies. In Machine Learning and Cybernetics, 2006 International Conference on, pages 3222-3227. IEEE, 2006. 